Volume 8, No. 7, July - August 2017

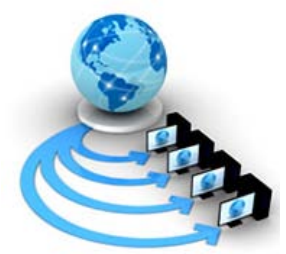

International Journal of Advanced Research in Computer Science

RESEARCH PAPER

\author{
Available Online at www.ijarcs.info
}

\title{
OPTIMIZING LEACH USING HYBRID ACO/PSO FOR MOBILE SINK IN WSNs: A REVIEW
}

\author{
Pallavi Sharma \\ Research Scholar \\ Computer Science and Engineering \\ DAV Institute of Engg \&technology \\ Jalandhar, India
}

\author{
Rajesh Kochher \\ Assistant Professor \\ Information Technology \\ DAV Institute of Engg \&technology \\ Jalandhar, India
}

\author{
Varsha \\ Assistant Professor \\ Information Technology \\ CT Group of Institutions, Shahpur \\ Jalandhar, India
}

\begin{abstract}
WSN consist of base station called sink and battery enabled sensor nodes. The energy of sensors is used to transmit data to the base station and it reduces with time. The energy consumption can also be reduced by using the idea of mobile sink which communicate between end user and sensor nodes. Later on concept of rendezvous nodes (RZ) and mobile sink was combined with LEACH to reduce energy consumption. In order to contribute more toward this direction Ant Colony Optimization (ACO) has been added to find the optimized route. The proposed work uses the concept of Hybrid ACO/PSO technique (finds the shortest route) have been used in optimizing the network lifetime and to improve energy efficiency.
\end{abstract}

Keywords - WSNs (Wireless Sensor Networks), LEACH, RZ (Rendezvous Node), ACO (Ant Colony Optimization), PSO (Particle Swarm Optimization).

\section{INTRODUCTION}

The WSN [6,8] comprises of vast amount of sensor nodes and a Sink or Base Station (BS) situated under the area of examination. Sensors are placed in random fashion i.e. with no planning in the region under examination, so in this case to be able to communicate with entire network they must adjust themselves means they must configured themselves. The nodes transmit the sensed information to BS, where data is being processed. In WSNs, major function of base station is to sends beacons (queries to nodes) to nodes. After getting the queries from base station, nodes sends the data switch back to BS. So BS act as gateway to internet i.e. data gathering and transmission of just applicable information to client by means of internet is finished by the base station. In single-hop transmission, every sensing element node transmits information via single hop to the BS i.e. by long distance transmission. The limitation of this method is that it is not effective in terms of energy consumption. So as to overcome this limitation, we should reduce the distance and this can be achieved by MultiHop Transmission. In Multi-Hop transmission every sensor node[16] transmit its information via different nodes, which in turn transmit that data to BS, there -by reduces the distance of transmission and consumption of energy once different node pretend as routers. WSNs are that, because the capability of every individual device node is restricted, the collective power of the whole network is adequate for the obligatory task. The Main elements of a WSN node are:-Controller, Communication device(s), Sensors/actuators, Memory ,Power offer. The key intention of graded routing or cluster primarily based routing is to apply maintain the energy usage of device nodes by connecting them in multi-hop communication among an exacting cluster. Cluster construction is often supported the energy reserve of sensors and sensors closeness to the Cluster Head (CHs). Agglomeration plays a key role for power saves in WSNs. Energy consumption, period of the network and quantifiability will be increased. Since solely cluster head node per cluster is crucial to perform routing task and therefore the former device nodes simply forward their knowledge to cluster head. Agglomeration has main applications in high-density device networks, as a result of its greatly easier to manage cluster of cluster representatives (cluster head) from every group than to manage complete device nodes. In WSNs[19] the device nodes square measure resource controlled which suggests they need restricted energy, transmit power, memory, and process capabilities. Energy consumed by the device nodes for human activity knowledge ranging from device nodes to the bottom station are that the central reason behind energy exhaustion in device nodes.

\section{RELATED WORK}

WSNs [19] consist of large number of sensor nodes and a sink or base station placed in the area of sampling. The sensors sense the data and send it to the BS. Then data is being processed at BS. If required, the BS can send the commands to nodes. Earlier data was transmitted by single hop long distance transmission from sensor nodes to BS [13].

Transmitting data through long distance was considered as not effective in terms of consumption of energy. Reducing the distance helps to reduce consumption of energy; thereby enhances network lifetime [12]. In order to overcome the 
drawback of single hop transmission, the solution was to use the concept of multi hop routing. In multi hop routing data was transmitted through other nodes, which will forward the data to BS; thereby reducing the distance of transmission of data and energy consumed by making the other nodes to behave as routers. The limitation of multi hop transmission was that the energy of nodes situated closer to BS will drops quickly.

So to overcome this drawback clustering can be the solution. In it members of cluster transmit their data to cluster head $(\mathrm{CH})$, while data collected by $\mathrm{CH}$ is transmitted to the BS.

LEACH protocol is an efficient adaptive clustering protocol [17]. LEACH protocol consist of two phases i.e. setup and steady phase. In setup phase clusters are formed and sensors transmit the sense information to local $\mathrm{CH}$. Then data collected at $\mathrm{CH}$ is firstly aggregated (i.e. by removing redundant data) and then transmitted to BS. LEACH[7] is based on the concept of random rotation for electing $\mathrm{CH}$, which in turn leads to even distribution of energy among sensors nodes because if there is no rotation of $\mathrm{CHs}$, the energy of $\mathrm{CH}$ will be quickly consumed and will die; thereby ending the ability of its member nodes to communicate in the network. LEACH uses time division multiple access (TDMA) in order to find when sensors can send the sensed data. It uses adaptive clustering, means nodes themselves decide to which cluster it belongs [15].

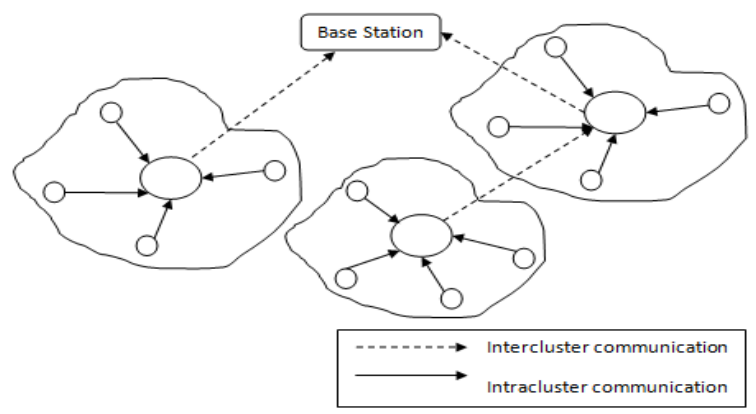

Fig.1. Data communication in a clustered network

In terms of energy consumption the LEACH works effectively than existing approaches. This is because of its rotation of $\mathrm{CH}$ and adaptive clustering.

The concept of mobile sink (MS) is an alternative technique that helps in minimizing the consumption of energy. The movement of MS [2,5] is either inside or around the trajectory for collecting data from nodes. The movement of sink is of two types i.e. controlled or uncontrolled. MS movement in predefined trajectory is known as controlled movement. While when MS moves randomly in the given area then such movement is known as uncontrolled movement. By making the sink closer to nodes helps in reducing the distance of transmission, thereby enhances the energy efficiency and network lifetime of WSNs.

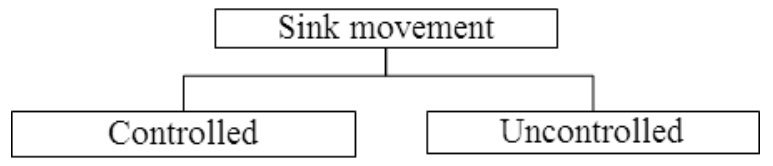

Fig.2. Movement of Sink
Since MS cannot be closer to all the nodes for collecting data. So the concept of Rendezvous Point (RP) has been developed. It is usually located closer to the path of Mobile Sink. When MS comes closer to RN, it will pass the data [16]. The signals send by MS are known as beacons, which notifies the RN about MS arrival.

Later on in the wireless sensor network, RZ Leach is enhanced by adding the concept of ant colony optimization algorithm for finding the optimized route for transmitting data. Finding the optimized route helps in reducing the distance of transmission thereby helps in decreasing consumption of energy thereby prolongs lifetime of network.

Proposed system uses the concept of one hop field neural network in the selection process of cluster head and then clusters are formed by using an optimized LEACH protocol. After cluster formation process hybrid ACO/PSO route optimization technique has been applied to find the shortest route to transmit the data, which in turn results into reduction of transmission distance and thus enhances the network lifetime.

\section{CLUSTERING TECHNIQUES}

\subsection{LOW ENERGY ADAPTIVE CLUSTERING HIERARCHY (LEACH)}

In Routing Low-Energy Adaptive Clustering Hierarchy (LEACH) is the first hierarchical based protocol [1]that uses irregular rotation of local cluster base stations (BS). LEACH is used when a node in the network crash or the battery stops working. It is a self-organizing and an adaptive clustering protocol where nodes are grouped into clusters and each cluster contains Cluster Head $(\mathrm{CH})$ and a Cluster Member (CM). The CHs are not chosen statically in a network as the sensor nodes may die rapidly. LEACH uses irregular protocol to balance the energy utilization for the nodes by separating the Cluster heads role to other nodes. Furthermore, to monitor the channel access in a cluster LEACH uses TDMA (Time Division Multiple Access) protocol. CHs have the authority to assign TDMA [9] slots to the cluster members. $\mathrm{CH}$ and $\mathrm{CM}$ communicate peer-to-peer through the time period that has been given to that member while other members are in a sleep state resulting in reduce in energy recreation.

\section{Characteristics of LEACH:}

- Creation of clusters

- Information aggregation

- communication.

- $\quad$ Random Death of nodes.

\section{Assumptions of LEACH:}

- $\quad$ All nodes have the concept of initial energy.

- Omni-directional antenna is used.

- Sink is repaired and remote for wsn.

- Energy consumption.

LEACH has the two phases.

(a) Setup phase, 
(b) Steady phase

\section{Setup Phase:}

In starting phase, every node arrive at conclusion to become cluster-head $(\mathrm{CH})$ in the ongoing round .While Selecting $\mathrm{CH}$; every node produce arbitrary number between 0 and 1.The limit is supposed for the current round and accomplished arbitrary number is set towards the limit of given round . if quantity is less than the threshold $\mathrm{T}(\mathrm{n})$ that node will chose as $\mathrm{CH}[4,10,18]$ for the current round.

The threshold is set up as:

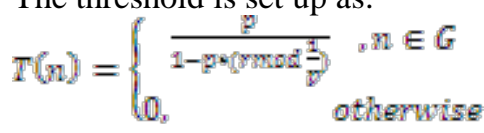

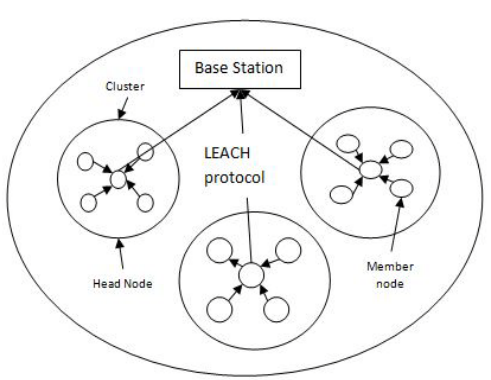

Figure3: LEACH protocol

The ratio of cluster-head nodes in the total number nodes is equal to ' $\mathrm{p}$ '.

$r$ is the present round number.

$\mathrm{G}$ is the set of nodes that have been choose as $\mathrm{CH}$ nodes in the former $1 / p$ rounds.

The cluster head $[11,17,20]$ generates a TDMA schedule for different nodes within its cluster which tell which node can transmit at which time. It permits the radio element of lower cluster head to switched off uniformly rather than its transmission time.

\section{Steady phase:}

This operation is divided into frames, in fact where the nodes transfer their information to cluster-head for the ultimate part one frame in their sign time slit. The cluster-head moves the whole information to the Base place (BS).

In this project, it chooses the cluster-head arbitrarily and the whole energy load of system is simultaneously expand to every alarm node by which it can consume less energy and their for enhance the system life. LEACH routing protocol is much better in the form of minimum transmission energy and strong sign, in the static clustering algorithm. When LEACH sweeps and neither nodes allow to handle on information from base place nor native with the worldwide network .To increase the system life time, the leach offers much better development and reduce the total amount of information but it has some circumstances:

1) It may use same $\mathrm{CH}$ node again in another round because .It has more energy.
2) It appear a hotspot problem, i.e. the $\mathrm{CH}$ employs more energy than usual alarm nodes

3) The special alarm node creates duplicate information that trouble on other cluster-head

4) The $\mathrm{CH}$ are chosen randomly and the node which have less energy are selected as cluster-head than that node could not able to successfully load more energy. It results an ancient end of life of these node and decrease the network life time.

\section{CLUSTER SELECTION}

Once all CHs and RN [3,14] are selected, using CSMA protocol CHs and RNs broadcasts a message to Normal Nodes (NN). At this stage to hear the message broadcasted by CHs and RNs, the receivers of NNs must be 'ON'. The NNs themselves decide their cluster and find $\mathrm{RN}$ closer to it. Depending upon the distance from CHs and RNs, this decision is made. Signal strength helps to determine the distance i.e. more the signal strength, lesser is the distance means $\mathrm{NN}$ will attach to that cluster head having lesser distance.

\section{Data transmission}

Once the creation and establishment of schedule has been done, the next step is to find the shortest routes for data transmission with the purpose of reducing utilization of energy. So Hybrid ACO/PSO optimization technique has been applied. Firstly Ant Colony Optimization (ACO) technique has been applied to find the shortest route from node to sink. The probability of finding the optimized route is given by formula

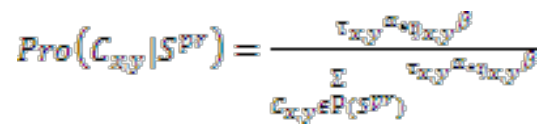

where, $S^{p y}$ is partial solution, $\mathrm{P}$ is set of all the paths from the city $\mathrm{x}$ to all adjacent cities still not visited by ant, $C_{\mathrm{z} y}$ is the path from the city $x$ to $y$, Pro is the probability, $\tau_{x y}$ is the amount of pheromone in the path $C_{X y}, 5_{\mathrm{Xg}}$ is the heuristic factor usually $D_{X Y}=\frac{Q}{d_{x y}}$, where $d_{X y}$ be the distance along the cities $\mathrm{x}$ and $\mathrm{y}, \mathrm{Q}$ be the some constant and ' $\alpha$ ' and ' $\beta$ ' are algorithm parameters

After getting the set of results of shortest routes, particle swarm optimization (PSO) is applied to get refine the results. It provides a population based search technique that uses personal experience (Pbest), overall experience (Gbest) and the current position of particle to find the coming location in the area of search. The PSO algorithm works as given below.

- $\quad$ Set parameters $\mathrm{w}_{\min ,} \mathrm{w}_{\max }, \mathrm{C} 1$ and $\mathrm{C} 2$ (ranges from 2 to 2.05) of PSO.

- Initialize population of particle having population ' $\mathrm{P}$ ' (usually varies from 10 to 100) and velocities 'Vel'(initial velocity is $10 \%$ of position).

- $\quad$ set iteration $i=1$

- $\quad$ calculate fitness of particle $F_{x}^{i}=f\left(P_{y}^{i}\right)$, find index of best particle $b$

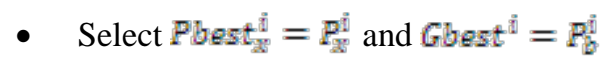


- $w=w_{\max }-i *\left(w_{\max }-w_{\mathrm{min}}\right) / M a x i t$ where, Maxit varies from 500 to 10000

- $\quad$ Update $\mathrm{V}$ and position of particle

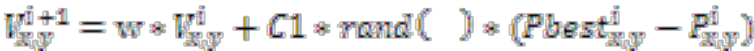
$\mathrm{V}_{\mathrm{x}, \mathrm{y}}^{\mathrm{i}+1}=\mathrm{w}^{*} \mathrm{~V}_{\mathrm{x}, \mathrm{y}}^{\mathrm{i}}+\mathrm{C} 1^{*} \operatorname{rand}()^{*}\left(\right.$ Pbest $\left._{\mathrm{x}, \mathrm{y}}^{\mathrm{i}}-\mathrm{P}_{\mathrm{x}, \mathrm{y}}^{\mathrm{i}}\right)+\mathrm{C} 2 *$ $\operatorname{rand}()^{*}\left(\right.$ Gbest $\left._{\mathrm{x}, \mathrm{y}}^{\mathrm{i}}-\mathrm{P}_{\mathrm{x}, \mathrm{y}}^{\mathrm{i}}\right)$

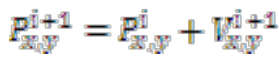

- $\quad$ Evaluate fitness $F_{z^{i}+1}^{i+1}=f\left(P_{z^{i}}^{i+1}\right)$, find index of best particle b1

- $\quad$ Update Pbest if $F_{z i}^{i+1}<F_{i s}^{i 1}$ Then Pbest $t_{z}^{i+1}=P_{z}^{i+1}$ else Pbest ${ }_{\mathrm{z}}^{i+1}=$ Pbest $_{\mathrm{z}}^{i}$

$$
\text { Gbest }^{i+1}=\text { Pbest }^{i+1}, \mathrm{~b}=\mathrm{b} 1 \text {, else Gbest }{ }^{i+1}=\text { Gbest }^{i}
$$

- If $i<$ Maxit then $i=i+1$ and goto step 6 else goto step 12

- $\quad$ Print Gbest $t^{\mathfrak{D}}$

After finding the shortest routes, transmission of data starts. Assuming that in all rounds nodes have some data and all nodes generates data at the same rate. While during this phase the receivers of all CHs and RNs must kept 'ON'. After getting data from all the nodes, $\mathrm{CH}$ starts aggregating the data gathered (i.e. removes redundant data) and passed it either to mobile sink or to the closest RN.

\section{CONCLUSION}

Ant Colony Optimization (ACO) is being widely used in optimizing the network routing protocols. Ant Based Routing can play a significant role in the enhancement of network life time. In this dissertation, Inter-cluster ACO-PSO algorithm for routing of data packets in the network and an attempt has been made to minimize the efforts wasted in transferring the redundant data sent by the sensors which lie in the close proximity of each other in a densely deployed network. This work has focused on evaluating the performance of rendezvous nodes based LEACH protocol. The overall goal is to find the effectiveness of the rendezvous nodes based LEACH when ACO -PSO inter-cluster data aggregation is applied on it.

\section{V.REFERENCES}

[1] Mottaghi, saeid, and mohammad reza zahabi. "optimizing leach clustering algorithm with mobile sink and rendezvous nodes." aeu-international iournal of electronics and communications 69, no. 2: 507-514, 2015.

[2] Yu gu; inf. Syst. Archit. Sci. Res. Div., nat. Inst. Of inf ; yusheng ji;jie li;baohua zhao. "efficient scheduling for the mobile sink in wireless sensor networks with delay constraint”,ieee trans parallel distrib system;24(july(7)):pp 131020. İeee tokyo, japan, 2013.

[3] Konstantopoulos, c; dept. Of inf., univ. Of piraeus, piraeus,; pantziou, g.; gavalas, damianos; mpitziopoulos, a. "a rendezvous-based approach enabling energy-efficient sensory data collection with mobile sinks in:ieee trans parallel distrib system;23(may(5)):pp 809-17. İeee, greece ,2013.
[4] Khalid hussain, abdul hanan abdullah, khalid m. Awan, faraz ahsan and akhtab hussain," cluster head election schemes for wsn and manet: a survey",world applied sciences journal 23 (5): 611-620, issn 1818-4952, 2013.

[5] Wang liu; dept. Of comput. Sci., univ. Of sci. \& technol. Of china, hefei; kejie lu; jianping wang ; guoliang xing, "performance analysis of wireless sensor networks with mobile sinks" ieee trans veh technol;61(july(6)):2777-88 . İeee, china , 2012.

[6] Reetika munjal, bhavneesh malik "approach for improvement in leach protocol for wireless sensor network", second international conference on advanced computing \& communication technologies (ieee); pp.517-521.ieee, rohtak, haryana 7-8 jan 2012.

[7] Meenakshi sharma and anil kumar shaw "transmission time and throughput analysis of eee leach, leach and direct transmission protocol: a simulation based approach”, advanced computing: an international journal ( acij ),vol.3, no.5. September 2012.

[8] Singhal, s., gankotiya,a., kumar, a., shikha,v. "an investigation of wireless sensor network: a distributed approach in smart environment"ieee second international conference on advanced computing \& communication technologies,pp.522-529.ieee, july 2012.

[9] Peng, j., chengdong, w., yunzhou, z. And fei, c., "a low-energy adaptive clustering routing protocol of wireless sensor networks”, ieee international conference on wireless communications, networking and mobile computing (wicom), september, pp. 1-4. İeee, 2011 .

[10] K.ramesh and dr. K.somasundaram ," a comparative study of cluster head selection algorithms in wireless sensor networks", international journal of computer science \& engineering survey (ijcses), november vol.2, no.4., 2011.

[11] Melese, d. G., xiong, h., and gao q., "consumed energy as a factor for cluster head selection in wireless sensor networks", ieee 6th international conference on wireless communications networking and mobile computing (wicom), september ,pp. 14.ieee, 2010

[12] Weifa liang; sch. Of comput. Sci., australian nat. Univ., canberra, act, australia ; jun luo;xu xu. "prolonging network lifetime via a controlled mobile sink in wireless sensor networks" in: global telecommunication conference (ieee globecom 2010), pp.1-6. Australia , december 2010.

[13] Zhengj,jamalipoura.wirelesssensornetworksanetwor kingperspective.wiley-ieeepress;2009.

[14] Guoliang xing;city univ. Of hong kong, kowloon ; tian wang;zhihui xie;weijia jia."rendezvous planning in mobilityassisted wireless sensor networks" trans mob comput ; pp 143043. Hong kong, 2008.

[15] Kumar,g.s;dept. Of comput. Sci., cochin univ. Of sci. \& technol., cochin ; vinu paul,m.v;jacob k.p. "mobility metric based leach-mobile protocol“ in: $1^{\text {th }}$ international conference on advanced computing and communication,.cochin, 2008. Pp 24853.

[16] Liu b ,bras p,dousse o,nain p, "mobility improves coverage of sensor networks" in:proceedings of the $6^{\text {th }}$ acm international symposium on mobile ad hoc networking and computing, 2005. Pp.300-8.

[17] Heinzelman,w.r;mit,cambridge,ma,usa;chandrakasan,a;balakrish nan,h."energy-efficient communication protocol for wireless microsensor networks" in:ieee international conference on system sciences , usa, 2000. Pp10-20.

[18] Nidhi bhatia, manju bala and varsha, "cluster head selection using qos strategy in wsn", international interdisciplinary conference on science, technology, engineering, management , pharmacy and humanities, singapore.

[19] Upasana, manju bala,varsha sahni, "fuzzy logic based ccwm approach to improve qos \& energy efficiency in wsn", international journal of modern embedded system, volume 4, issue 6, december, 2016, issn: 2320-9003(online), 21-26.

[20] Jaspinder kaur, varsha sahni “ energy efficient linear cluster handling protocol for wsn” international journal of computational science, information technology and control engineering, singapore. Vol.3, no.1/2, april. 